\title{
Perfil das notificações de câncer relacionado ao trabalho em um hospital referência em oncologia no estado de Mato Grosso do Sul, Brasil
}

\author{
Profile of work-related cancer notifications in a referral \\ hospital in oncology of the Mato Grosso do Sul state, Brazil
}

\author{
Perfil de notificaciones de cáncer relacionado con el trabajo \\ en un hospital de referencia en oncología en el estado de \\ Mato Grosso do Sul, Brasil
}

\author{
Jackeline Lazorek Saldanha da Silva ${ }^{1}$ \\ Luciana Contrera ${ }^{2}$ \\ Elen Villegas Campos ${ }^{3}$
}

Alexandra Maria Almeida Carvalho ${ }^{4}$

${ }^{1}$ Mestre em Enfermagem pela Universidade Federal de Mato Grosso do Sul (UFMS). Especialista em Enfermagem de Unidade de Terapia Intensiva pela Universidade para o Desenvolvimento do Estado e da Região do Pantanal (UNIDERP). Graduada em Enfermagem pela Universidade do Estado de Mato Grosso (UNEMAT). Enfermeira do Hospital de Câncer Alfredo Abrão. E-mail: jacke_lazorek@hotmail.com, ORCID: https://orcid.org/0000-0003-2272-2787

${ }^{2}$ Doutora em Doenças Infecciosas e Parasitárias pela Universidade Federal de Mato Grosso do Sul (UFMS). Mestre em Enfermagem pela Universidade Estadual de Campinas (UNICAMP). Graduada em Licenciatura Plena em Enfermagem e Obstetrícia pela Universidade Federal de São Carlos (UFSCar). Graduada em Enfermagem pela UFSCar. Professora associada do Programa de Pós-Graduação Mestrado Acadêmico em Enfermagem do Instituto Integrado de Saúde da UFMS. E-mail: lucontrera@gmail.com, ORCID: https://orcid.org/0000-0002-8751-0817

${ }^{3}$ Doutoranda em Saúde e Desenvolvimento na Região Centro-Oeste, na Universidade Federal de Mato Grosso do Sul (UFMS). Mestre em Saúde e Desenvolvimento na Região Centro-Oeste pela UFMS. Especialista em Enfermagem do Trabalho pela Universidade Católica Dom Bosco (UCDB).

Especialista em Gestão do Trabalho e da Educação em Saúde pela Universidade Federal do Rio Grande do Norte (UFRN). Graduada em Enfermagem pela UFMS. Professora do curso de Medicina da Universidade Estadual de Mato Grosso do Sul (UEMS).E-mail: elenvillegas@hotmail.com, ORCID: http://orcid.org/0000-0002-6751-0131

\footnotetext{
${ }^{4}$ Doutora em Biosystems Engineering and Environmental Science, pela Universidade do Tennessee. Mestre em Engenharia Elétrica pela Universidade Federal de Uberlândia

(UFU). Graduada em Engenharia Civil pela Universidade Estadual Paulista Júlio de Mesquita Filho (UNESP). Professora adjunta da Universidade Federal de Mato Grosso do Sul (UFMS) e coordenadora do Programa de Pós-Graduação Saúde e Desenvolvimento na Região Centro-Oeste. Atualmente desenvolve projetos em saúde ambiental, saúde do trabalhador e avaliação de serviços de saúde. E-mail: profalexandraufms@gmail.com,

Orcid: http://orcid.org/0000-0002-7868-3214
} 
Resumo: A Organização Mundial da Saúde (OMS) estimou que, no ano de 2030, são esperados 27 milhões de casos de câncer, 17 milhões de mortes e 75 milhões de pessoas com câncer. Uma em cada seis mortes é relacionada à doença, que apresenta grande impacto em trabalhadores. Este estudo teve o objetivo de analisar as fichas de notificação de câncer relacionado ao trabalho em um hospital de referência em oncologia no estado de Mato Grosso do Sul. Metodologia: estudo quantitativo que analisou fichas de notificação de câncer relacionado ao trabalho (anos de 2015-2017) em um hospital oncológico. Foram analisadas 41 fichas de notificação por meio de estatística descritiva. Resultados: 85,4\% eram do gênero masculino; 87,7\%, com idade superior a 50 anos (39,0\% de 51 a 70 anos e 48,8\% acima de 70 anos); 51,2\%, da raça branca; 83\%, autônomo; e 62,4\%, com ocupação rural. O câncer mais notificado foi de pele não melanoma $(56,1 \%)$ e o de boca, faringe, esôfago e estômago (21,9\%).

Palavras-chave: trabalho; câncer; notificação.

\begin{abstract}
The World Health Organization (WHO) has estimated that, in the year 2030, 27 million cancer cases, 17 million deaths, and 75 million people with cancer are expected. One in six deaths is related to the disease that has the greatest impact on workers. This study aimed to analyze the notification forms of cancer-related to work in a referral hospital in oncology of the Mato Grosso do Sul state, Brazil. Methodology: a quantitative study that analyzed notification forms of work-related cancer (years 2015-2017) in an oncology hospital. Forty-one notification forms were analyzed using descriptive statistics. Results: $85.4 \%$ were male; $87.7 \%$ over 50 years old (39.0\% between 51 and 70 years old and $48.8 \%$ over 70 years old); $51.2 \%$ of the white race; $83.0 \%$ freelancer; and $62.4 \%$ with rural occupation. The most notified cancer was of non-melanoma skin (56.1\%) and the mouth, pharynx, esophagus, and stomach (21.9\%).
\end{abstract}

Keywords: work; cancer; notification.

Resumen: La Organización Mundial de la Salud (OMS) ha estimado que, en el año 2030, se esperan 27 millones de casos de cáncer, 17 millones de muertes y 75 millones de personas con cáncer. Una de cada seis muertes está relacionada con la enfermedad que tiene un gran impacto en los trabajadores. El objetivo de este estudio fue analizar las formas de notificación del cáncer relacionado con el trabajo en un hospital de referencia en oncología en el estado de Mato Grosso do Sul. Metodología: estudio cuantitativo, que analizó las formas de notificación del cáncer relacionado con el trabajo (años 2015-2017) en un hospital de oncología. Se analizaron 41 formularios de notificación utilizando estadísticas descriptivas. Resultados: el 85,4\% eran hombres; 87,7\% mayores de 50 años (39,0\% entre 51 y 70 años y $48,8 \%$ mayores de 70 años); 51,2\% de la raza blanca; 83,0\% trabajadores no registrados; y $62,4 \%$ con ocupación rural. El cáncer más notificado fue el de la piel no melanoma (56,1\%) y el de boca, faringe, esófago y estómago $(21,9 \%)$.

Palabras clave: trabajo; cáncer; notificación. 
Perfil das notificações de câncer relacionado ao trabalho em um hospital referência em oncologia no estado de Mato Grosso do Sul, Brasil

\section{INTRODUÇÃO}

Câncer é o nome dado a um conjunto de mais de 100 doenças que têm em comum o crescimento desordenado de células, as quais invadem tecidos e órgãos. As células normais do organismo sofrem uma alteração no seu DNA e passam a receber instruções erradas para as suas atividades (INCA, 2019a).

As alterações podem ocorrer em genes especiais, denominados proto-oncogenes, que a princípio são inativos em células normais. Quando ativados, os proto-oncogenes tornam-se oncogenes, responsáveis por transformar as células normais em células cancerosas (INCA, 2019a).

No mundo, o câncer é a segunda principal causa de morte, sendo responsável por 9,6 milhões de mortes em 2018. Em nível global, uma em cada seis mortes são relacionadas à doença. O impacto econômico dessa doença foi estimado em aproximadamente US\$1,16 trilhão (OPAS, 2001).

A Organização Mundial da Saúde (OMS) relatou que, no ano 2030, são esperados 27 milhões de casos de câncer, 17 milhões de mortes e 75 milhões de pessoas com câncer. O maior efeito desse aumento será em países de baixa e média renda (ONCOGUIA, 2015).

No Brasil, estima-se, para cada ano do triênio 2020-2022, a ocorrência de 625 mil casos novos de câncer. A distribuição da incidência por região geográfica mostra que a Região Sudeste concentra mais de $60 \%$ da incidência, seguida pelas Regiões Nordeste (27,8\%) e Sul (23,4\%) (INCA, 2019b).

Os tipos de câncer mais frequentes em homens, à exceção do câncer de pele não melanoma, serão próstata $(29,2 \%)$, cólon e reto $(9,1 \%)$, pulmão (7,9\%), estômago $(5,9 \%)$ e cavidade oral $(5,0 \%)$. Nas mulheres, exceto o câncer de pele não melanoma, os cânceres de mama $(29,7 \%)$, cólon e reto $(9,2 \%)$, colo do útero $(7,4 \%)$, pulmão $(5,6 \%)$ e tireoide $(5,4 \%)$ figurarão entre os principais. O câncer de pele não melanoma representará 27,1\% de todos os casos de câncer em homens e 29,5\% em mulheres (INCA, 2019b).

O câncer tem causas externas, do meio ambiente (água, terra e ar/ ambiente de trabalho), e internas (fatores genéticos, hormônios, condições imunológicas e mutações genéticas), além do estilo e dos hábitos de vida. Isso explica por que algumas pessoas desenvolvem câncer e outras não, quando expostas a um mesmo carcinógeno (INCA, 2019a). 
Causas do meio ambiente incluem o ambiente de trabalho, em que vários agentes são descritos como relacionados ao câncer ocupacional, sendo alguns: exposição à radiação ultravioleta ou radiação ionizante (radiologia e usinas nucleares); o asbesto, encontrado em materiais como a fibra de amianto e o cimento; os hidrocarbonetos aromáticos, encontrados na fuligem; as aminas aromáticas, usadas na produção de tintas e pesticidas; e o benzeno, encontrado nas usinas siderúrgicas e nos solventes de tintas e colas (INCA, 2013).

Segundo o Ministério da Saúde estudos mais recentes atribuem a fatores ambientais e ocupacionais cerca de 19\% de todos os tipos de câncer (BRASIL, 2012).

O câncer relacionado ao trabalho é definido como decorrente da exposição a agentes carcinogênicos presentes no ambiente de trabalho, mesmo após a cessação da exposição. A legislação trabalhista exige a existência do nexo causal, ou seja, a relação inequívoca na qual a ocorrência da doença é devido à exposição aos agentes carcinogênicos aos quais o trabalhador está ou esteve exposto. Porém nem sempre isso é possível, visto que o câncer, muitas vezes, ocorre tardiamente (anos depois) (INCA, 2018).

Segundo o INCA (2018) o Registro de Câncer de Base Hospitalar (também conhecido como Registro Hospitalar de Câncer ou RHC) permite caracterizar a origem do câncer e facilitar as ações de vigilância em saúde.

Diante do exposto, este estudo tem o objetivo de analisar as fichas de notificação de câncer relacionado ao trabalho em um hospital de referência em oncologia no estado de Mato Grosso do Sul.

\section{MATERIAL E MÉTODOS}

Estudo quantitativo exploratório, transversal, com dados secundários, cujo objetivo foi analisar as fichas de notificação de câncer relacionado ao trabalho em um hospital de referência em oncologia no estado de Mato Grosso do Sul, no período de janeiro de 2015 a janeiro de 2017.

O hospital escolhido para o estudo é uma entidade filantrópica, beneficente, sem fins lucrativos, especializado em oncologia, cujo atendimento 
abrange 98\% do Sistema Único de Saúde. Suas ações são pautadas em diagnóstico, tratamento, reabilitação e prevenção do câncer.

As fichas de notificação de câncer são preenchidas pelo enfermeiro durante a consulta de enfermagem, em que ocorre a investigação do histórico ocupacional e possível relação com o câncer.

Para análise das fichas de notificação, foram definidos critérios de inclusão, tais como: fichas legíveis; dados completos; período de janeiro de 2015 a janeiro de 2017 e com dados de investigação de câncer relacionado ao trabalho. Foram também definidos como critérios de exclusão: fichas ilegíveis; com dados incompletos; que não tratavam de câncer relacionado ao trabalho; e fora do período estipulado.

Foi identificado um total de 60 fichas de notificação de câncer emitidas pelo hospital, entre janeiro de 2015 e janeiro de 2017; porém, após verificação dos critérios de inclusão e exclusão, foram retiradas 19 notificações, resultando em uma amostra final de 41 fichas de notificação.

Os dados das notificações foram tabulados em planilha Excel, com as seguintes variáveis de estudo: sexo; idade; etnia/raça; escolaridade; atividade remunerada; profissão; tabagismo e tempo de exposição ao tabagismo; exposição e tempo de exposição a agentes cancerígenos; tipo de câncer.

O projeto de pesquisa foi aprovado pelo Comitê de Ética em Pesquisa em Seres Humanos da Universidade Federal de Mato Grosso do Sul, mediante o parecer n. 1844052.

\section{RESULTADOS}

Do total de 41 notificações analisadas, 85,4\% eram do sexo masculino, sendo $87,8 \%$ com idade superior a 50 anos (39,0\% de 51 a 70 anos e 48,8\% acima de 70 anos); 51,2\%, da raça branca; 61\% não informaram a escolaridade e, entre os que informaram, constatou-se que $17,1 \%$ estudaram da 1a a 4 a série incompletas; $83 \%$ exerciam atividade de trabalho informal, conforme Tabela 1. 
Tabela 1 - Caracterização das fichas de notificação de câncer relacionado ao trabalho, Hospital do Câncer, Mato Grosso do Sul, 2015-2017

\begin{tabular}{lcc}
\hline \multicolumn{1}{c}{ Dados pessoais } & N. & $\%$ \\
\hline Sexo & 35 & \\
Masculino & 6 & 85,4 \\
Feminino & & 14,6 \\
Faixa etária & 5 & \\
De 30 a 50 anos & 16 & 12,2 \\
De 51 a 70 anos & 20 & 39,0 \\
Acima de 70 anos & & 48,8 \\
Raça & 9 & \\
Sem informação & 21 & 22,0 \\
Branca & 1 & 51,2 \\
Negra & 2 & 2,4 \\
Amarela & 8 & 4,9 \\
Parda & & 19,5 \\
Escolaridade & 25 & \\
Sem informação & 4 & 61,0 \\
Analfabeto & 7 & 9,7 \\
1a a 4á série incompletas & 2 & 17,1 \\
5a a 8a série incompletas & 1 & 4,9 \\
Ensino fundamental completo & 2 & 2,4 \\
Ensino médio completo & & 4,9 \\
Situação no mercado de trabalho & 1 & 2,4 \\
Sem informação & 3 & 7,3 \\
Empregado com carteira assinada & 34 & 7,3 \\
Autônomo & 3 & \\
Aposentado & & \\
\hline
\end{tabular}

Fonte: Elaborado pelo autor com base nos dados da pesquisa (2015-2017).

Em relação ao tabagismo $(n=41)$, obteve-se $36,6 \%$ de fumantes e $19,5 \%$ de ex- fumantes, o que resultou em $56,1 \%$ ( $n=23$ ) de indivíduos expostos ao tabaco ao longo da vida; desses, $69,5 \%$ relataram que o tempo de exposição ao tabaco era superior a 24 anos (Tabela 2).

Todas as fichas de notificação relataram exposição a um ou mais agentes cancerígenos, no entanto houve maior porcentagem de exposição à 
radiação ionizante (68,3\%); seguida por exposição a óleos minerais (24,4\%); benzeno (17,1\%); alcatrão, hidrocarbonetos alifáticos e sílica (14,6\% cada um); asbesto/amianto, cromo e hormônios (4,9\% cada um); composto de níquel e radiação ionizante ( $2,4 \%$ cada um). O tempo predominante de exposição a agentes cancerígenos foi de 25-36 anos (26,8\%) (Tabela 2).

Tabela 2 - Número de fichas por câncer relacionado ao trabalho, segundo variáveis de estudo, 2015 a 2017

\begin{tabular}{|c|c|c|c|}
\hline Variáveis & Total de Fichas & $\mathbf{N}$. & $\%$ \\
\hline Tabagismo & 41 & & \\
\hline Sem informação & & 8 & 19,5 \\
\hline Sim & & 15 & 36,6 \\
\hline Ex-fumante & & 8 & 19,5 \\
\hline Não & & 10 & 24,4 \\
\hline Tempo de exposição ao tabaco & 23 & & \\
\hline Sem informação & & 2 & 8,7 \\
\hline Até 12 anos & & 1 & 4,4 \\
\hline De 13 a 24 anos & & 4 & 17,4 \\
\hline De 25 a 36 anos & & 7 & 30,4 \\
\hline De 37 a 48 anos & & 5 & 21,7 \\
\hline Acima de 48 anos & & 4 & 17,4 \\
\hline Agentes* & 41 & & \\
\hline Radiação não ionizante & & 28 & 68,3 \\
\hline Óleos minerais & & 10 & 24,4 \\
\hline Benzeno & & 7 & 17,1 \\
\hline Alcatrão & & 6 & 14,6 \\
\hline Hidrocarbonetos alifáticos & & 6 & 14,6 \\
\hline Sílica & & 6 & 14,6 \\
\hline Asbesto/Amianto & & 2 & 4,9 \\
\hline Cromo & & 2 & 4,9 \\
\hline Compostos de níquel & & 1 & 2,4 \\
\hline Radiação ionizante & & 1 & 2,4 \\
\hline Outros & & 13 & 31,7 \\
\hline Tempo de exposição aos agentes & 41 & & \\
\hline Até 12 anos & & 6 & 14,6 \\
\hline De 13 a 24 anos & & 7 & 17,1 \\
\hline
\end{tabular}




\begin{tabular}{lccc}
\hline \multicolumn{1}{c}{ Variáveis } & Total de Fichas & N. & \% \\
\hline De 25 a 36 anos & 11 & 26,8 \\
De 37 a 48 anos & 9 & 22,0 \\
Acima de 48 anos & 8 & 19,5 \\
\hline
\end{tabular}

*Um ou mais agentes por paciente.

Fonte: Elaborado pelo autor com base nos dados da pesquisa (2015-2017).

Houve predomínio de câncer de pele (não melanoma), com 56,1\% das notificações ( $n=41)$; seguido por câncer de boca, faringe, esôfago e estômago (21,9\%); e câncer de pulmão ou mediastino (12,2\%). Em relação à profissão, predominaram os trabalhadores rurais, com 62,4\% (n=41); pedreiros, com 15,5\%; frentista de posto, com 4,5\%; e o restante distribuiu-se de acordo com a Tabela 3.

Tabela 3 - Número de fichas por câncer relacionado ao trabalho, segundo tipos de câncer e ocupação, 2015 a 2017 (n=41)

\begin{tabular}{lcc}
\hline \multicolumn{1}{c}{ Variáveis } & N. & \% \\
\hline Tipos de câncer & 23 & 56,1 \\
Pele (não melanoma) & 9 & 21,9 \\
Boca, faringe, esôfago e estômago & 5 & 12,2 \\
Pulmão e mediastino & 2 & 4,9 \\
Pele (melanoma) & & \\
Ocupação* & 1 & 2,2 \\
Caminhoneiro & 1 & 2,2 \\
Carpinteiro & 1 & 2,2 \\
Construtor & 2 & 4,5 \\
Frentista & 1 & 2,2 \\
Indústria asfáltica & 1 & 2,2 \\
Indústria de petróleo & 1 & 2,2 \\
Mestre de obras & 1 & 2,2 \\
Motorista & 1 & 2,2 \\
Operador de máquina & 7 & 15,5 \\
Pedreiro & 28 & 62,4 \\
Trabalhador rural & & \\
\hline
\end{tabular}

*Algumas notificações têm mais de uma profissão.

Fonte: Elaborado pelo autor com base nos dados da pesquisa (2015-2017). 


\section{DISCUSSÃO}

\subsection{Perfil sociodemográfico: sexo e idade}

O perfil das pessoas que foram atendidas no Hospital do Câncer, descrito nas fichas de notificação, revelou a predominância do sexo masculino (85,4\%), trabalhadores autônomos $(83,0 \%)$ e rurais $(62,4 \%)$, com faixa etária acima dos 70 anos $(48,8 \%)$ e com escolaridade inferior a 4 anos de estudo (1 1 a e 4a séries não completas, $17,1 \%$ das fichas).

Segundo o Boletim Epidemiológico da Saúde do Trabalhador do Paraná, observa-se que o sexo masculino apresenta a maior proporção dentro da somatória das notificações de agravos ocupacionais (54\%). Tal fato é explicado pela Organização Internacional do Trabalho (OIT), que relatou que os homens exercem, em maior número, funções em ramos produtivos de risco, como na construção civil, por exemplo, e expostos a substâncias que são cancerígenas ou causadoras de doenças circulatórias e respiratórias (CEST-PR, 2017; OIT, 2016).

Considerando as notificações por câncer de pele, que, neste estudo, totalizaram 61\%, Pires et al. (2018), ao avaliarem o perfil sociodemográfico de pacientes diagnosticados com câncer de pele por meio de um serviço de dermatologia, encontraram também um percentual de 50\% de indivíduos do sexo masculino, sendo a maioria com idade entre 50 e 80 anos.

A idade elevada nesses casos é esperada, visto que o dano causado pelos agentes cancerígenos é cumulativo, levando a lesões que provocam o câncer. A idade elevada foi também encontrada por Simoneti et al. (2016), na cidade de São Paulo, em que a idade destacada foi superior a 60 anos, com 71,6\% da amostra dos diagnósticos de câncer.

\subsection{A relação da escolaridade com a exposição a riscos ocupacionais}

Em 2009, a ocupação informal no país absorvia 43.816.696 trabalhadores e se concentrava, principalmente, nas atividades rurais, que ocupavam o primeiro lugar, seguidas dos serviços domésticos e da construção civil (ARAÚJO; LOMBARDI, 2013). 
Araújo e Lombardi (2013) indicaram que trabalhadores com menos escolaridade estão inseridos em trabalhos informais, incluindo os autônomos; e, ao contrário, os com maior escolaridade encontram-se no setor formal, sendo que, destes, $70 \%$ das mulheres e $53 \%$ dos homens tinham cursado pelo menos o ensino médio, e, no informal, $27 \%$ dos homens e $18 \%$ das mulheres relataram somente até 3 anos de estudo.

A menor escolaridade está diretamente ligada à menor qualificação profissional, e estes trabalhadores, segundo Wünsch Filho et al. (2008), estão submetidos a maiores riscos de exposição a substâncias químicas, fumaça de diesel, tintas, corantes e outros agentes cancerígenos.

No Brasil, há predominância de pequenos proprietários de terra associados à agricultura familiar. Isso se relaciona com o resultado deste estudo, em que $62,4 \%$ declararam ser trabalhadores rurais. O trabalhador rural tem maior vulnerabilidade aos cânceres ocupacionais, devido à exposição ao sol (câncer de pele) e exposição a agrotóxicos; além disso, a orientação em relação ao uso dos produtos é incipiente e não há fiscalização em relação à utilização adequada de Equipamentos de Proteção Individual (EPIs) (MOURA et al., 2018).

Os trabalhadores rurais recebem orientação específica para determinados tipos de agrotóxicos e utilizam o mesmo parâmetro para outros que são mais concentrados, o que acarreta erros quantitativos prejudiciais ao homem, ao ambiente e à cultura submetida. Na região do Vale do São Francisco, cerca de $21 \%$ das indicações de uso dos agrotóxicos, feitas pelos vendedores, diferem do preconizado pelo Ministério da Pecuária, Agricultura e Abastecimento (MOURA et al., 2018).

\section{AGENTES CANCERÍGENOS}

Os agentes cancerígenos são aqueles que têm relação com a causa do câncer. A seguir, será descrita a relação dos agentes cancerígenos como possível causa de alguns tipos de cânceres descritos neste trabalho.

\subsection{Agente: radiação - não ionizante e ionizante}

A radiação não ionizante é aquela que tem energia capaz de mover átomos em torno de uma molécula, mas não é capaz de remover elétrons; 
como exemplo, a luz solar é a energia emitida pelo sol na forma de radiação eletromagnética não ionizante (INCA, 2010).

A radiação ionizante é capaz de remover elétrons dos átomos, criando os íons. Pode ser encontrada na forma de partículas ou ondas eletromagnéticas. Como exemplo, pode-se citar as partículas alfa, beta, raios gama, raios $X$ e nêutrons. Este tipo de radiação é utilizado em tratamento de radioterapia e diagnósticos, por meio de radiografia, tomografia, mamografia e mapeamento com radiofármacos (INCA, 2010).

As radiações, sejam ionizantes ou não, estão relacionadas ao câncer de pele, sendo a radiação solar classificada no grupo I pela International Agency for Research on Cancer, com evidência suficiente de carcinogenicidade em seres humanos (IARC, 1997b).

Há dois tipos de câncer de pele: não melanoma (carcinomas basocelular e espinocelular) e o melanoma maligno. O não melanoma surge nas células basais ou nas escamosas, e o melanoma tem origem nos melanócitos, células que produzem a melanina, o pigmento que dá cor à pele. O não melanoma representa 95\% do total dos casos de câncer de pele (ANTÔNIO CÂNDIDO DE CAMARGO CANCER CENTER, [s.d.]).

O pior prognóstico para melanomas está associado à idade superior a 60 anos, gênero masculino, lesões localizadas no tronco, tumores de maior espessura e padrão socioeconômico mais baixo (SOUZA; FISCHER; SOUZA, 2004; BALZI et al., 1998).

Entre os fatores de risco para o câncer de pele estão: pessoas de pele clara, com cabelos ruivos ou loiros; trabalhadores de atividades agrícolas; e exposição excessiva ao sol (SBCD, 2019). A cor clara da pele, neste estudo, foi relatada em $51,2 \%$ das fichas.

O câncer de pele (não melanoma) foi o mais descrito nas fichas de notificação, constando em 56,1\% das fichas. Este tipo de câncer de pele é o mais frequente no Brasil e apresenta altos percentuais de cura, se for detectado e tratado precocemente (INCA, 2018). Já o melanoma foi citado em $4,9 \%$ das fichas.

Segundo o Ministério da Saúde o câncer não melanoma está relacionado à ocupação ao ar livre, como o trabalho rural e da construção civil, por 
exemplo, que foram citados neste estudo, sendo 68,3\% e 19,9\% das fichas, respectivamente (BRASIL, 2012).

O alto número de trabalhadores rurais com câncer de pele apresenta nexo causal com a exposição não ionizante e também pode estar relacionado à exposição a agrotóxicos (JOBIM, et al., 2010), visto que muitos destes trabalhadores não fazem uso de EPIs, segundo estudo de Recena e Caldas (2008), em Culturama, MS, que demonstrou que agricultores não usavam luvas, máscaras ou roupas impermeáveis, embora conhecessem esses equipamentos e considerassem que devessem usá-los. Eles apresentavam, ainda, exposição a riscos ocupacionais por tempo superior a 8 horas por dia, acentuando o risco de desenvolver câncer.

Este estudo apresentou alto número de notificações de câncer de pele $(56,1 \%)$, o que pode estar relacionado ao trabalho rural e à cor clara da pele.

\subsection{Agente: alcatrão}

A exposição ao alcatrão foi relatada em 14,6\% das fichas. Percivall Pott, em 1975, descreveu a relação do alcatrão com o câncer de pele por exposição ocupacional. O câncer foi localizado no escroto de trabalhadores limpadores de chaminés, devido ao contato direto da pele com fuligem. Anos mais tarde (1915), Yamagiwa \& Ichikawa descrevem a ocorrência de tumores de pele em animais, pela aplicação de alcatrão do carvão diretamente sobre a pele deles, confirmando o alcatrão como agente cancerígeno (BRASIL, 2001).

Neste estudo, a associação do câncer de pele com o alcatrão não é conclusiva, visto que as profissões com exposição ocupacional ao alcatrão foram descritas em apenas duas fichas de notificação (4,4\%), sendo indústria asfáltica e de petróleo. Para uma associação mais clara, seria necessária uma investigação mais profunda sobre dados da exposição.

Porém essa investigação é difícil devido ao período de latência (tempo decorrido entre o início da exposição ao carcinógeno e a detecção clínica do tumor). Esse período pode ser de 20 a 50 anos, o que dificulta o nexo causal entre a exposição e a doença, particularmente no caso dos cânceres relacionados ao trabalho (BRASIL, 2001). 
No entanto o alcatrão pode ser fortemente associado ao alto índice de câncer de boca, faringe, esôfago e pulmão (34,1\% das fichas). Segundo a International Agency for Research on Cancer (IARC) (1990), o alcatrão é uma mistura com cerca de 4.000 compostos químicos que se forma durante a combustão incompleta dos materiais orgânicos presentes nos cigarros.

A frequência de fumantes ativos relatada nas fichas foi de $36,6 \%$, e tabagismo pregresso (ex-tabagistas), 19,5\%, sendo a exposição ao alcatrão superior a 25 anos (30,4\% das fichas). O tabagismo está entre os principais fatores de risco para o câncer, sendo responsável por $90 \%$ dos casos de câncer de pulmão e por 30\% das mortes ocorridas por outros tipos de câncer, como boca, laringe, esôfago, estômago, pâncreas, fígado, rim, bexiga, colo do útero e leucemia (MOURA, 2018).

Neste estudo, o alto índice de sujeitos expostos ao alcatrão do cigarro, ao longo da vida, pode ser relacionado ao fato de que a amostra se constitui predominantemente em sexo masculino, de baixa renda, e trabalhadores rurais. Dados recentes mostram que os homens têm uma prevalência maior relacionada ao tabagismo, além de um consumo maior em pessoas de baixa renda. Observa-se prevalência do tabagismo em trabalhadores manuais, manutenção e atividades agrícolas (MALTA et al., 2015).

Além disso, o tabagismo aumenta o fator de risco ocupacional, pois o fumo interage com algumas substâncias, aumentando sua capacidade cancerígena (INCA, 2005).

\subsection{Agente: sílica e amianto}

Sílica refere-se aos compostos de dióxido de silício, representada pelo símbolo SiO2. É um mineral duro e o mais abundante na crosta terrestre; encontra-se em rochas e areias. As três formas de sílica cristalina são: quartzo, trimidita e cristobalita (NIOSH, 2002).

Já o amianto, também denominado asbesto, é uma forma fibrosa dos silicatos minerais. Compõe-se de silicatos hidratados de magnésio, ferro, cálcio e sódio (INCA, 2010).

As profissões com exposição à sílica exercem atividades nas indústrias de cerâmica (tijolo, telha, porcelana, olaria, refratários e vitrificados); 
indústrias de cimento; construção civil; construção naval e extração mineral. Já o amianto é muito utilizado na confecção de caixas d'água, telhas, tubulações, tintas, revestimentos e isolamentos térmicos e acústicos, lonas e pastilhas, além de produtos têxteis, como tecidos especiais que oferecem resistência mecânica, química (INCA, 2010).

Segundo o INCA (2018), as profissões com exposição à sílica e ao amianto relatadas neste estudo foram: construtor (2,2\%), mestre de obras $(2,2 \%)$, pedreiro $(15,5 \%)$ e agricultor $(62,4 \%)$. Trabalhadores da construção civil se expõem ao amianto ao fixarem as telhas de cimento-amianto com parafusos e ao inalar a poeira de amianto (BRASIL, 2001).

O Brasil é o quinto maior produtor de amianto, com produção em cerca de 237 mil toneladas por ano, na indústria do cimento, com exportação direta de 70 mil toneladas por ano (GIANNASI, 1997).

Segundo a IARC, da OMS, a sílica cristalina está classificada como Grupo 1, reconhecidamente cancerígena para seres humanos, e o amianto, como agente cancerígeno sem limite seguro para exposição (IARC,1997a).

A poeira de sílica cristalina, quando inalada, pode se acumular nos alvéolos, lesionar os brônquios e provocar fibrose pulmonar, que é responsável pela diminuição da complacência pulmonar que prejudica o processo de trocas gasosas. Os sintomas são: tosse e falta de ar progressiva (INCA, 2010).

A mortalidade por câncer de pulmão tem risco duas a três vezes maior nos trabalhadores expostos à sílica após o controle por outros fatores, como fumo (INCA, 2010).

As doenças decorrentes da exposição à sílica, em especial a silicose e o câncer de pulmão, são objetos de notificação nacional ao Ministério da Saúde, regulamentada pela Portaria n. 777/GM, de 28 de abril de 2004, que dispõe sobre os procedimentos técnicos para a notificação compulsória de agravos à saúde do trabalhador em rede de serviços sentinela específica no Sistema Único de Saúde (SUS).

Já o amianto está relacionado à ocorrência de asbestose, que é muito semelhante à silicose, com quadro de inflamação e fibrose pulmonar. Além de estar associada ao aparecimento de câncer (pulmão e trato gastrointestinal) e de mesotelioma (tumor raro e de difícil diagnóstico) (OPAS, 2001). 
Entre as fichas de notificação, 14,6\% relataram exposição à sílica e 4,9\% ao amianto, podendo esta estar relacionada ao câncer de pulmão (12,2\% das fichas).

Desde 1955, é reconhecida a relação causal entre a exposição ao asbesto ou amianto e a ocorrência dos mesoteliomas da pleura, do peritônio e do câncer de pulmão (OPAS, 2001).

Estudos de prevalência de asbestose e silicose são escassos no Brasil. Estudo realizado com 86 trabalhadores da indústria de fibrocimento, com mais de 10 anos de exposição ao asbesto; destes, 25\% dos trabalhadores apresentavam asbestose (INCA, 2010).

Já Rodel Speger (1995), estudando os efeitos do amianto do tipo anfibólio, observou que este tipo de asbesto oferece um risco cinco vezes maior para câncer de pulmão do que outros tipos, fato confirmado anos depois pela WHO (1998), que afirmou que o amianto está relacionado a diversas formas de doença pulmonar (asbestose, câncer pulmonar e mesotelioma de pleura e peritônio) (CASTRO, 2003).

O uso do amianto é proibido na Europa e em outros países da América Latina, já no Brasil é proibido apenas o uso de amianto do tipo anfibólio, e a pulverização (spray) de qualquer amianto proíbe também o trabalho de menores de 18 anos nas áreas de produção (CASTRO, 2003).

Outro câncer muito notificado nas fichas foi o de boca, faringe, laringe e estômago (21,9\%), sendo o de boca, laringe e faringe relacionado pelo INCA (2019b) a agrotóxicos e ao amianto, e o de estômago e do esôfago, à exposição a poeiras da construção civil.

\subsection{Agentes: óleos minerais}

O óleo mineral provém da destilação do petróleo bruto em junção com a pressão atmosférica, que, com a mistura de diversos hidrocarbonetos, origina o óleo mineral (SILVA, 2011).

O óleo mineral é muito utilizado na indústria automobilística, na lubrificação de máquinas, equipamentos, peças e em processo de usinagem. Está presente também em graxas e vaselinas, assim como adjuvantes em produtos agrícolas, como herbicidas e inseticidas (OLIVEIRA; OLIVEIRA; MELO, 2003). 
A exposição aos óleos minerais foi relatada em $24,4 \%$ das fichas de notificação. Segundo o INCA (2019b), este câncer está relacionado ao câncer de estômago e esôfago e, também, ao câncer de pele não melanoma, muito frequente neste estudo (56,1\%).

No Brasil, a Norma Regulamentadora n. 15, do Ministério do Trabalho e Emprego, caracteriza como insalubridade de grau máximo, de modo geral, a manipulação de óleos minerais, sem especificar o tipo; para tanto, é preconizado para trabalhadores expostos o uso de aventais para proteção do tronco e luvas impermeáveis (BRASIL, 1978).

\subsection{Agentes: cromo e níquel}

As primeiras evidências da ação cancerígena do cromo datam de 1948, sendo ele incluído no grupo 1 dos agentes cancerígenos pela IARC, em 1990. Estudos epidemiológicos têm mostrado relação entre a exposição dos trabalhadores da indústria de produção de cromatos e a ocorrência de câncer de pulmão (IARC, 1990). Já o níquel foi considerado cancerígeno desde 1952, também incluído no grupo 1. Uma publicação de 1990 sobre a ação cancerígena do níquel, descrevendo os resultados de nove estudos de coorte e um caso-controle, revelou consistente aumento da incidência de câncer de pulmão e nasal entre indivíduos expostos a este metal (TERRA FILHO; KITAMURA, 2006).

Terra Filho e Kitamura (2006) citaram que as profissões expostas ao cromo e ao níquel estão envolvidas na fabricação de bicromados, aço inox, baterias, indústria gráfica, produção de refratários, fundição de zinco, fabricação de revestimento de superfícies (cromeação) e atividades de pintura a revólver (spray).

Das fichas, 4,9\% tiveram relato de exposição ao cromo e 2,4\% ao níquel, podendo se relacionar ao câncer de pulmão (12,2\% das fichas).

\subsection{Agentes: hidrocarbonetos alifáticos - benzeno, tolueno e xileno}

A exposição a hidrocarbonetos alifáticos (benzeno, tolueno e xileno) foi relatada em $14,6 \%$ das fichas, e a exposição exclusivamente ao benzeno foi relatada em 17,1\% das fichas de notificação de câncer. 
O benzeno é um hidrocarboneto aromático, volátil, inflamável, com odor característico, que, nas Condições Normais de Temperatura e Pressão (CNTP), apresenta-se sob a forma líquida e incolor (INCA, 2010).

É uma substância reconhecida pela OMS como carcinogênica, sem limite seguro de exposição, e é um composto usado principalmente na indústria de petróleo (gasolina, hexano, querosene, tolueno), inseticidas, solventes e removedores de tintas (verniz), siderúrgicas e metalúrgicas (INCA, 2010).

O benzeno está relacionado principalmente às leucemias em trabalhadores, segundo o INCA. Este tipo de câncer não foi identificado nas fichas de notificação, sendo os cânceres identificados sem relação com a exposição ao benzeno (INCA, 2018).

Já o xileno e o tolueno não são considerados carcinogênicos pela IARC (1990), portanto não serão discutidos neste estudo.

\section{CONSIDERAÇÕES FINAIS}

Em decorrência de fatores ocupacionais, o câncer de maior notificação foi de pele não melanoma, seguido pelo câncer de boca, faringe, esôfago e estômago, pulmão e mediastino e, por último, pele (melanoma).

O agente de maior exposição ocupacional foi a radiação ionizante a qual estão expostos os trabalhadores rurais, profissão predominante neste estudo.

Este estudo teve como limitação o baixo número de fichas de notificação, que, segundo o Centro de Referência de Saúde do Trabalhador (CEREST, 2017) de Mato Grosso do Sul (MS), ocorre devido a uma subnotificação das fichas; além disso, foi identificada uma baixa qualidade no preenchimento das fichas.

Ações voltadas ao treinamento da equipe de captação desses dados deveriam ser realizadas, a fim de que os profissionais que participam deste processo compreendessem a importância da captação correta dos dados nos formulários.

Estudos com foco investigativo sobre o câncer ocupacional relacionando o trabalho, agentes cancerígenos ao qual o trabalhador foi exposto e o câncer notificado são de extrema importância para nortear ações de prevenção ao câncer nessa população que move o país. 


\section{REFERÊNCIAS}

ANTÔNIO CÂNDIDO DE CAMARGO CANCER CENTER [A.C. CAMARGO CANCER CENTER]. Pele não melanoma. A.C CAMARGO, São Paulo, [s.d.]. Disponível em: https://www.accamargo.org.br/sobre-o-cancer/tipos-de-cancer/pele-naomelanoma. Acesso em janeiro de 2019.

ARAÚJO, A. M. C.; LOMBARDI, M. R. Trabalho informal, gênero e raça no Brasil do início do século. Revista Cadernos de Pesquisa, São Paulo, v. 43, n. 149, p. 452477, ago. 2013.

BALZI, D.; CARLI, P.; GIANNOTTI, B.; BUIATTI, E. Skin melanoma in Italy: a populationbased study on survival and prognostic factors. European Journal of Cancer, [s.l.], v. 34, n. 5, p. 699-704, 1998.

BRASIL. Ministério do Trabalho e Emprego. NR 15 - atividades e operações insalubres. Brasília: Ministério do Trabalho e Previdência Social, 1978.

BRASIL. Ministério da Saúde. Diretrizes para a vigilância do câncer relacionado ao trabalho. Brasília-DF: Ministério da Saúde, 2012.

CASTRO, H.; GIANNASI, F.; NOVELLO, C. A luta pelo banimento do amianto nas Américas: uma questão de saúde pública. Ciência e Saúde Coletiva, São Paulo, v. 8, n. 4. p. 903-11. 2003.

CENTRO DE REFERÊNCIA EM SAÚDE DO TRABALHADOR [CEREST]. Vigilância Epidemiológica em Saúde do Trabalhador. Campo Grande, 12 abr., 2017. (Boletim Informativo).

CENTRO ESTADUAL DE SAÚDE DO TRABALHADOR [CEST]. Boletim epidemiológico da saúde do trabalhador do Paraná: notificações dos agravos da saúde do trabalhador no Paraná (2006 a 2016). Curitiba: CEST, 2017.

INTERNATIONAL AGENCY FOR RESEARCH ON CANCER [IARC]. Silica, some silicates, coal dust and para-aramid fibrils. Lyon: IARC, 1997a.

INTERNATIONAL AGENCY FOR RESEARCH ON CANCER [IARC]. Solar and Ultraviolet Radiation. Lyon: IARC, 1997b.

INTERNATIONAL AGENCY FOR RESEARCH ON CANCER [IARC]. Chromium and chromium compounds. Chromium [VI] (Group 1). Metallic chromium and chromium [III] compounds (Group 3). Lyon: IARC, 1990. p. 49. 
Perfil das notificações de câncer relacionado ao trabalho em um hospital referência em oncologia no estado de Mato Grosso do Sul, Brasil

INSTITUTO NACIONAL DE CÂNCER JOSÉ ALENCAR GOMES DA SILVA [INCA]. O que é câncer? Brasília: INCA, 2019a.

INSTITUTO NACIONAL DE CÂNCER JOSÉ ALENCAR GOMES DA SILVA [INCA]. Estimativa 2020: incidência de câncer no Brasil. Rio de Janeiro: INCA, 2019b.

INSTITUTO NACIONAL DE CÂNCER JOSÉ ALENCAR GOMES DA SILVA [INCA]. Câncer de pele não melanoma. Rio de Janeiro: INCA, 2018.

INSTITUTO NACIONAL DE CÂNCER JOSÉ ALENCAR GOMES DA SILVA [INCA]. O câncer e seus fatores de risco: o que a educação pode evitar? 2. ed. rev. atual. Rio de Janeiro: INCA, 2013.

INSTITUTO NACIONAL DE CÂNCER JOSÉ ALENCAR GOMES DA SILVA [INCA]. Coordenação de Prevenção e Vigilância. 2. ed. rev. atual. Rio de Janeiro: INCA, 2010.

INSTITUTO NACIONAL DE CÂNCER JOSÉ ALENCAR GOMES DA SILVA [INCA]. Vigilância do câncer ocupacional e ambiental. Rio de Janeiro: INCA, 2005.

JOBIM, P. F. C.; NUNES, L. N.; GIUGLIANI, R.; CRUZ, I. B. M. Existe uma associação entre mortalidade por câncer e uso de agrotóxicos? Uma contribuição ao debate. Revista Ciência e Saúde Coletiva, Rio de Janeiro, v. 15, n. 1, p. 288-10, 2010.

MALTA, D. C.; VIEIRA, M. L.; SZWARCWALD, C. L.; CAIXETA, R., BRITO, S. M. F.; REIS, A. A. C. Tendência de fumantes na população brasileira segundo a Pesquisa Nacional de Amostra de Domicílios 2008 e a Pesquisa Nacional de Saúde 2013. Revista Brasileira de Epidemiologia, São Paulo, v. 18, n. 2, p. 45-56, 2015.

MOURA, L. T. R.; ANINGER, P. R. L. C.; BARBOSA, A. V.; BEDOR, C. N. G. Caracterização epidemiológica de trabalhadores com câncer em uma região de fruticultura irrigada. Revista Baiana de Saúde Pública, Bahia, v. 42, n. 1, p. 7-25, 2018.

NATIONAL INSTITUTE FOR OCCUPATIONAL SAFETY AND HEALTH [NIOSH]. Hazards review: health effects of occupational exposure to respirable crystalline silica. Cincinnati: NIOSH, 2002.

OLIVEIRA, C. P.; OLIVEIRA, C. A. L.; MELO, W. J. Efeito da adição de óleos mineral e vegetal a acaricidas no controle do ácaro-da-leprose dos citros Brevipalpus phoenicis. Revista Brasileira de Fruticultura, Jaboticabal, v. 25, n. 2, p. 224-6, 2003. 
ONCOGUIA. Estimativas de câncer no mundo. Oncoguia, Rio de Janeiro, 2015. Disponível em: http://www.oncoguia.org.br/conteudo/estimativas-nobrasil/1705/1/. Acesso em: 1o jan. 2018.

ORGANIZAÇÃO INTERNACIONAL DO TRABALHO [OIT]. Segurança e saúde no trabalho para homens e mulheres. Genebra: OIT, 2016.

ORGANIZAÇÃO PAN-AMERICANA DA SAÚDE [OPAS]. Doenças relacionadas ao trabalho: manual de procedimentos para os Serviços de Saúde série A. Brasília: OPAS, 2001.

GIANNASI, F.; THEBAUD-MONY, A. Occupational exposure to asbestos in Brazil. International Archives of Occupational and Environmental, [s.I.], v. 3, n. 2, p. 1507, 1997.

PIRES, C. A. A.; FAYAL, A. P.; CAVALCANTE, R. H.; FAYAL, S. P.; SANTOS, M. A. L. Câncer de pele: caracterização do perfil e avaliação da proteção solar dos pacientes atendidos em serviço universitário. Journal of Health Biological Sciences, Belém, v. 6, n. 1, p. 54-59, 2018. doi: http://dx.doi.org/10.12662/2317-3076jhbs.v6i1.1433. p54-59.2018

RECENA, M. C. P.; CALDAS, E. D. Percepção de risco, atitudes e práticas no uso de agrotóxicos entre agricultores de Culturama, MS. Revista de Saúde Pública, São Paulo, v. 42, n. 2, p. 294-301, 2008.

SILVA. A. E. O. Transposição didática: a química dos óleos lubrificantes. 2011. Trabalho de conclusão da disciplina TCC 2 (Graduação em Química) - Universidade de Brasília, Instituto de Química, Brasília-DF, 2011.

SIMONETI, F.; CUNHA, L. O.; GOMES, C. T. V.; NOVO, N. F.; PORTELLA, D. L.; GONELLA, H. A. Perfil epidemiológico de pacientes com tumores cutâneos malignos atendidos em ambulatório de cirurgia plástica de serviço secundário no interior de São Paulo. Revista da Faculdade Ciência Médica, São Paulo, v. 18, n. 2, p. 98-102, 2016.

SOCIEDADE BRASILEIRA DE CIRURGIA DERMATOLÓGICA [SBCD]. Câncer de pele. São Paulo: SBCD, 29 ago. 2019.

SOUZA, S. R. P.; FISCHER, F. M.; SOUZA, J. M. P. Bronzeamento e risco de melanoma cutâneo: revisão da literatura. Revista Saúde Pública, São Paulo. v. 38, n. 4, p. 58898. 2004. 
Perfil das notificações de câncer relacionado ao trabalho em um hospital referência em oncologia no estado de Mato Grosso do Sul, Brasil

TERRA FILHO, M.; KITAMURA, S. Câncer pleuropulmonar ocupacional. Jornal Brasileiro de Pneumologia, São Paulo, v. 32, n. supl. 2, p. S60, 2006.

WÜNSCH FILHO, V.; ANTUNES, J. L. F.; BOING, A. F.; LORENZI, R. L. Perspectivas da investigação sobre determinantes sociais em câncer. Physis, Rio de Janeiro, $v$. 18, n. 3, p. 427-50, 2008.

WORLD HEALTH ORGANIZATION [WHO]. Environmental Health Criteria 203: chrysotile asbesto. Geneva: WHO, 1998. [Relatório]. 
Jackeline Lazorek Saldanha da SILVA; Luciana CONTRERA; Elen Villegas CAMPOS 